\title{
Rezension: Eliane Welte, Information der Öffentlichkeit über die Tätigkeit der Strafjustiz
}

Dominique Strebel *

Die Strafjustiz muss sich erklären. Zum einen verlangen dies Bundesverfassung (Art. 30 Abs. 3 BV) und Strafprozessordnung (Art. 69 StPO), zum andern ist es ein Gebot der Zeit: Die Gerichtsberichterstattung wird in den Medienhäusern abgebaut, und in Social Media dominieren weder journalistisch noch juristisch ausgebildete Personen die Diskussion. Die Dissertation von Eliane Welte zieht daraus wichtige Schlüsse: Erstens muss die Strafjustiz ihr originäres Produkt - das Urteil, den Strafbefehl oder die Einstellungsverfügung - niederschwellig und unkompliziert zugänglich machen - zum Beispiel mit einer Liste der erlassenen Endentscheide in einer zentralen Datenbank im Internet. Zweitens muss die Strafjustiz auch über sich als Institution aktiv kommunizieren: Sie soll auf öffentliche Kritik reagieren und sich bemühen, Verständnis für ihre Anliegen zu wecken. Dafür braucht es Medienbeauftragte und Kommunikationskonzepte. Die Dissertation zeigt umfassend, welche Formen der Berichterstattung (von Print über TV bis zu Live-Ticker aus dem Gerichtssaal) zu welchen Spannungen mit den Kommunikationsanliegen der Strafjustiz führen. Und wie sie damit optimal umgeht.

Zitiervorschlag: Dominique Strebel, Rezension: Eliane Welte, Information der

Öffentlichkeit über die Tätigkeit der Strafjustiz, in: sui-generis 2017, S. 314

URL: $\quad$ sui-generis.ch/52

DOI: $\quad$ https://doi.org/10.21257/sg.52

* Dominique Strebel, lic. iur., ist Studienleiter an der Schweizer Journalistenschule MAZ und CoHerausgeber der Zeitschrift medialex. 
1 In den letzten zehn Jahren hat sich bei vielen Gerichten in Sachen Öffentlichkeitsarbeit ein Gesinnungswandel vollzogen: Es gilt nicht mehr der Grundsatz, dass eine Justiz nur dann gut ist, wenn man nicht über sie spricht. Im Gegenteil - die Justiz hat begonnen, ihre Urteile mit Medienmitteilungen aktiv zu kommunizieren und der Öffentlichkeit ihre Tätigkeit zu erklären (etwa an einem Tag der offenen Tür). Verschiedene Gerichte haben Medienbeauftragte eingestellt und Kommunikationskonzepte erarbeitet (etwa in Luzern, am Ober- und Bezirksgericht Zürich sowie am Bundesgericht). Im gleichen Zeitraum hat das höchste Schweizer Gericht in einigen Leitentscheiden das verfassungsmässige Gebot der Justizöffentlichkeit (Art. 30 Abs. 3 BV) aktualisiert für eine Zeit, in welcher im Strafrecht der Strafbefehl die erdrückend dominante Form der Verfahrenserledigung, und das öffentlich verhandelte Urteil das Exotikum darstellt. ${ }^{1}$

So kommt die Dissertation von Eliane Welte zur richtigen Zeit und legt mit dem Strafrecht den richtigen Schwerpunkt. ${ }^{2}$ Sie spannt den Bogen vom Anspruch auf Zugang zu Urteilen, Strafbefehlen und Einstellungsverfügungen über die Analyse der Formen der medialen Berichterstattung (Print- und Online-Text, Fernsehbericht, Tweet, Facebook-Post und Live Ticker) bis hin zu den Grundsätzen einer tragfähigen Kommunikation der Strafjustiz. So entsteht ein umfassendes

\footnotetext{
Vgl. etwa BGE 137 I 16; Urteil des Bundesgerichts 1B_68/2012 vom 3. Juli 2012; Urteil des Bundesgerichts 1C_123/2016 vom 21. Juni 2016.

2 Für eine Zusammenfassung der wichtigsten Ergebnisse durch die Autorin: Eliane Welte, Strafjustiz vs. Medien und Öffentlichkeit - zwei Akteure mit gegensätzlichen Interessen, in: suigeneris 2017, 201.
}

Bild der Justizöffentlichkeit in ihrem ganzen Wirkungskontext. Und es werden Zusammenhänge sichtbar, die auch den zögerlichsten Richter davon überzeugen können, dass heutzutage kein Weg an aktiver Kommunikation vorbeiführt. Die Gedanken sind zwar an der Strafjustiz entwickelt, aber auf die Zivil- und Verwaltungsjustiz weitgehend übertragbar.

3 Im ersten Teil der Dissertation geht Welte dem Informationsanspruch der Medien und der Öffentlichkeit nach. Sie fasst die medienfreundliche Rechtsprechung des Bundesgerichts über weite Strecken treffend zusammen. In der Diskussion über die Reichweite des Anspruchs fehlen aber die Leitentscheide, in denen das Bundesgericht festgehalten hat, dass der Anspruch sich nicht nur auf das Dispositiv, sondern «auf das ganze Urteil mit Sachverhalt, rechtlichen Erwägungen und Dispositiv erstreckt.»3

4 Welte arbeitet für Strafbefehle eine zentrale Forderung schlüssig heraus. Bei dieser Form des Endentscheids besteht nämlich gemäss Bundesgericht zwar ein Anspruch auf Zugang, doch wird dieser in Realität unterlaufen, weil niemand weiss, dass die Strafbefehle überhaupt gefällt wurden. Daraus folgert die Autorin zu Recht: «Die blosse Auflage von Strafbefehlen bei der zuständigen Stelle kann nicht als zeitgemässe Umsetzung des Öffentlichkeitsprinzips im Bereich der Strafbefehle bezeichnet werden» (Seite 43). Sie fordert, dass in einer zentralen Datenbank im Internet eine Liste der erlassenen Strafbefehle publiziert wird. «Diese Entscheidlisten (...) sollten es interessierten Dritten ermöglichen,

3 BGE 139 I 129 E. 3.6; bestätigt in Urteil des Bundesgerichts 1C_123/2016 vom 21. Juni 2016. 
abzuschätzen, ob sich für sie die Einsichtnahme bei der zuständigen Staatsanwaltschaft lohnt» (Seite 44). Um dies zu erreichen, müssen die Entscheidlisten gemäss Welte die wichtigsten Eckpunkte des Strafbefehls enthalten: zuständige Staatsanwaltschaft, Tatbestände, Strafmass, aber vor allem auch eine Beschreibung des dem Beschuldigten zur Last gelegten Sachverhalts. Die dafür nötigen Anstrengungen der Behörden sind gemäss der Autorin «unumgänglich», damit die Kontrollfunktion der Öffentlichkeit nicht ausgehebelt wird (Seite 47). Zudem fordert Welte zu Recht einen voraussetzungslosen Anspruch auf Kenntnisnahme auch von Einstellungs- und Nichtanhandnahmeverfügungen, die ebenfalls in einer anonymisierten Liste unkompliziert und kostenlos im Internet einsehbar sein müssen. Sie regt auch an, die Aufzählung in Art. 69 Abs. 2 StPO um die Kategorie der Einstellungs- und Nichtanhandnahmeverfügungen $\mathrm{zu}$ ergänzen.

5 Den zweiten (mit rund 200 Seiten überwiegenden) Teil widmet die Autorin der aktuellen Informationslandschaft und der Zulässigkeit medialer Berichterstattung. Die Autorin holt weit aus, beginnt bei der Definition des Mediums, behandelt die Eigenarten von Presse, Rundfunk, Online-Medien und Social Media, die Interessenlagen der einzelnen Akteure und stellt die medialen Zwänge auf die Justiz dar. Dabei setzt sie die Bedeutung des Fernsehens zu hoch an, sinken doch auch dort die Quoten, während die Online-Nutzung vor allem via Social Media stark ansteigt.

6 Die auch juristisch anerkannten wichtigen Funktionen der Medien als Binde- glied zwischen Justiz und Bevölkerung (Information, aber auch Kontrolle und Kritik der Justiz), stellt Welte den negativen Effekten medialer Berichterstattung gegenüber und lotet so das Spannungsverhältnis zwischen Justiz und Medien aus. Dabei macht sie immer wieder interessante juristische Tiefenbohrungen wie zum Beispiel jene, ob es für die Umsetzung des Gebotes der Justizöffentlichkeit genüge, wenn die blosse Möglichkeit des Besuchs von Gerichtsverhandlungen besteht, auch wenn kaum jemand hingeht. Oder ob es im Gegenteil auch eine tatsächliche Kenntnisnahme der Entscheide braucht, die Justiz also gerade beim Verhandeln vor leeren Sitzbänken gefordert ist, ihre Urteile auf anderen Wegen der Bevölkerung zur Kenntnis zu bringen.

7 Der Echtzeitberichterstattung über TVBerichterstattung oder Live-Ticker steht die Autorin kritisch gegenüber. Sie zweifelt, ob diese Formen der Berichterstattung für den Nutzer jenseits der reinen Befriedigung der Sensationslust einen Mehrwert schafft. Welte weist darauf hin, dass das komplexe Geschehen vor Gericht mit diesen medialen Formen weder adäquat erklärt noch eingeordnet werden kann und medienethische sowie medienrechtliche Anforderungen schwer erfüllt werden können, weil keine Kontrollmechanismen dazwischen geschaltet sind. «Dieses Diktat der Aktualität verträgt sich mit den Zielen des Strafprozesses, nämlich der Aufklärung und Sanktionierung von Normverstössen in einem förmlichen, zuweilen langwierigen Verfahren, denkbar schlecht.» Deshalb bejaht Welte nicht nur das aktuelle Verbot von Radiound Fernsehaufnahmen von Gerichtsverhandlungen (Art. 71 Abs. 1 StPO), 
sondern fordert auch, dass die Strafprozessordnung mit einem Verbot textbasierter Live-Berichterstattung ergänzt wird.

8 Die massive Beschleunigung des Informationsprozesses kombiniert mit den Möglichkeiten von Social Media und Online-Kommentaren sieht die Autorin als grosse Herausforderung für die Öffentlichkeitsarbeit der Strafjustiz. Denn das umgehende Reagieren sei zu einem zentralen Faktor aller Medienarbeit geworden. "Auf diese Weise können sich im Internet auch Kritik und Unverständnis gegenüber der Strafjustiz wie ein Lauffeuer verbreiten.» Das Fazit der Autorin: Heutzutage reicht es nicht mehr aus, sich in Zurückhaltung zu üben und die Meinungsbildung über die Strafjustiz den Medien und der Bevölkerung zu überlassen.

9 Folgerichtig entwirft Welte im dritten Teil ihrer Dissertation das Konzept einer umfassenden Kommunikationsstrategie der Strafjustiz. Die Leitideen sind:

1. Information ist eine Bringschuld der Justiz. Die Justiz braucht also eine aktive Kommunikation: «Es reicht nicht mehr aus, sich in Zurückhaltung zu üben und die Meinungsbildung über die Strafjustiz den Medien und der Bevölkerung zu überlassen» (Seite 229).

2. Es genügt nicht, wenn die Justiz ihre Endentscheide veröffentlicht (aber bereits dies ist leider oft nicht gewährleistet A.d.V). Sie muss auf öffentliche Kritik reagieren und sich bemühen, Verständnis für ihre Anliegen zu wecken.
3. Die Kommunikation muss kontinuierlich gewährleistet sein. Dazu braucht es in der Regel Medienbeauftragte, die zeitlich flexibel erreichbar sind.

4. Die Öffentlichkeit fasst nur Vertrauen, wenn die Justiz auch zu ihren Fehlern steht (offene Fehlerkultur der Justiz).

10 Als Mittel der Kommunikation befürwortet die Autorin Medienmitteilungen, Medienkonferenzen, Treffen am Pressestamm, Tage der offenen Tür oder Interviews der Medienbeauftragten. Auskünften von Richtern gegenüber Medienschaffenden steht Welte skeptisch gegenüber, ohne dies vertieft zu begründen. Hier wäre eine Auseinandersetzung wünschenswert gewesen, um den Spielraum aufzuzeigen, den Richter haben - trotz Amtsgeheimnis, Gebot der Unabhängigkeit und Unschuldsvermutung. Denn das grösste Vertrauen schaffen noch immer konkrete Entscheidungsträger, die Red und Antwort stehen und so der Justiz ein Gesicht geben. Skeptisch ist Welte auch gegenüber Social Media. Die Spezifika dieser Plattformen würden sich nur zum Teil mit den Interessen der Strafjustiz decken, meint die Autorin. So zeichneten sich Social Media durch eher flüchtige und oberflächliche Kenntnisnahme der Inhalte aus. Gemäss Welte soll die Justiz über Social Media nicht in konkreten Dialog mit den Bürgerinnen und Bürgern treten. Da begibt sich die Autorin in ein Dilemma. Hat sie doch zuvor zu Recht festgestellt, dass sich «im Internet auch Kritik und Unverständnis gegenüber der Strafjustiz wie ein Lauffeuer verbreiten» kann. Deshalb wird eine moderne Kommunikation der Strafjustiz auf lange 
Sicht nicht darum herum kommen, auch in Social Media für Information und Korrektur ungerechtfertigter Kritik zu sorgen - wenn auch mit der gebotenen Zurückhaltung. Zudem eignen sich Social Media durchaus auch als Verbreitungskanal von Medienmitteilungen 4 .

11 So überzeugend Weltes Gesamtleistung ist, kommt das Juristische neben medienwirkungsanalytischen, medien- und kommunikationswissenschaftlichen Betrachtungen zuweilen etwas $\mathrm{zu}$ kurz. Gleichzeitig erscheinen die Betrachtungen über Ausrichtung, Publikationslogik und Rahmenbedingungen der Medien manchmal etwas oberflächlich. So verkürzt Welte Leistung und Ziel der Medien zu oft auf jene des Boulevardjournalismus: «Mit ihrer Berichterstattung über Strafverfahren geht es den Medien nur vordergründig um eine uneigennützige Informationsdienstleistung für das Publikum. Vielmehr erweist sich die Gerichtsberichterstattung für sie aus rein kommerziellen Motiven als höchst lukrativ» (Seite 10). Zwar mag dies für einen (wachsenden) Teil der Medien zutreffen. Doch bei meiner Arbeit für SRF, Beobachter, NZZ am Sonntag oder TagesAnzeiger habe ich keine übermässigen entsprechenden Tendenzen festgestellt. Das öffentliche Interesse an faktenbasierter Information der Öffentlichkeit, Relevanz und Justizkontrolle stand (fast) immer im Vordergrund.

Die Autorin hat auch ein etwas unbedarftes Bild von der journalistischen Arbeit. So beschreibt sie als Vorteil eines Interviews, dass Medienschaffende der interviewten Medienbeauftragten «die einzel- nen Fragen vorgängig» mitteilen (Seite 302). Kein guter Journalist wird dies tun. Und sie zitiert unkommentiert Literatur, die den Nutzen von Medienmitteilungen für Medienschaffende darin sieht, dass sie diese «ohne erneute aufwändige Überarbeitung veröffentlichen können» (Seite 299). Keine Journalistin, die ihr Handwerk beherrscht, wird so vorgehen. Sie wird den Text journalistisch aufbereiten - von der Verifikation bis zur Sprache.

13 Ein weiteres, grosses Plus des Buches sind die vielen konkreten Beispiele gelungener oder misslungener Kommunikation, welche die Autorin ausführlich analysiert - so etwa den Fall «Carlos» (als Musterbeispiel einer misslungenen Kommunikation) oder den NSU-Prozess in Deutschland (als Beispiel einer gelungenen Medienarbeit). So hat Welte ein eigentliches Standardwerk zur Justizkommunikation geschrieben, das jede Richterin und jeder Richter mit Gewinn lesen wird.

«Information der Öffentlichkeit über die Tätigkeit der Strafjustiz. Zur aktuellen Informationslandschaft und den Anforderungen an eine zeitgemässe Öffentlichkeitsarbeit der Strafbehörden», Eliane Welte, Schulthess Juristische Medien AG, Zürich 2016, ISBN: 978-3-7255-7475-9

4 So zum Beispiel der offizielle Twitter-Account des schweizerischen Bundesgerichts. 